\title{
Effects of squat amplitude on pelvic tilt and tibial inclination
}

\begin{abstract}
Strength training is commonly performed at two different knee flexion amplitudes: partial (to 90 degrees) or total (to 140 degrees). During these amplitudes, both the pelvis and the tibia are moved to ensure control of the center of gravity and displacement of the external overload. Forward or backward movement of the pelvic tilt may indirectly influence the internal load on the spine. Objective: To measure the effect of squat amplitude on pelvictilt and tibia inclination. Eighteen male subjects (age: $26 \pm 6$ years, height: $178 \pm 7 \mathrm{~cm}$, total body mass: $81.3 \pm 11 \mathrm{~kg}$, resistance training experience: $5 \pm 4$ years) were evaluated. Pelvic tilt and tibial inclination were measured by a digital inclinometer (Max Measure, USA, accuracy: $\pm 0.02^{\circ}$, resolution: $0.05^{\circ}$ ) during isometric squatting at partial and full amplitudes. The digital inclinometer was fixed on the sacrum and on the tibia, with aneutral spine position. A paired student t-test and a significance of $5 \%$ were used. There were significant differences in pelvic tilt between partial and full amplitudes $\left(+32.4^{\circ} \pm 10.9\right.$ and $-21.7^{\circ} \pm 12.3$, respectively, $\mathrm{P}<0.001)$. Maximum tibial inclination values were not significantly different between partial and total amplitudes (19.1 \pm 6.6 and $20.1 \pm 7.4$, respectively, $\mathrm{P}=0.225)$. It was concluded that the partial squat position produces anterior pelvic tilt while the full squat produces backward pelvic tilt. Inclination of the tibia is similar in both amplitudes of the squat.
\end{abstract}

Keywords: Exercise, posture, amplitude
Volume I Issue I - 2017

\author{
Paulo Henrique Marchetti,' Gustavo \\ ZorziFioravante,' Josinaldo Jarbas da \\ Silva, ${ }^{1,2}$ Willy Andrade Gomes, ${ }^{1,3}$ Enrico \\ Gori Soares,' Silvio Luiz Pecoraro,' \\ Fabio Sisconeto de Freitas,' Roberto \\ Aparecido Magalhães,' Marcelo Massatoshi \\ SenagaMiyatake,' Lee E Brown ${ }^{4}$ \\ 'Graduate Program in Science of Human Movement, Methodist \\ University of Piracicaba, Brazil \\ ${ }^{2}$ Physical Education Faculty, Brazil \\ ${ }^{3}$ Physical Education Faculty, Nove de Julho University \\ (UNINOVE), Brazil \\ ${ }^{4}$ Center for Sport Performance, Department of Kinesiology, \\ California State University, USA
}

Correspondence: Paulo H Marchetti, Methodist University of Piracicaba (UNIMEP), Graduate Program in Science of Human Movement, Brazil,Tel I3400-9| I,

Email dr.pmarchetti@gmail.com

\section{Introduction}

The squatexerciseis a multi-joint task, and canbeconsidereda fundamental exercise for lower body strength, general fitness, and rehabilitation. Several studies have shown that manipulating the amplitude ofthe squat exercise results in altered muscle activity ${ }^{1-3}$ however, research on pelvic movements in the squat are limited. ${ }^{4}$

Some research methodologies suggesta correct way to perform the squat, ${ }^{5}$ but the correct technique is still controversial, with suggestions that thelumbar curve should be maintained throughout the squat, ${ }^{6}$ where as others suggest avoiding a rounded lumbar spine. ${ }^{7}$ For heavy squats $^{8,9}$ suggest the squat should be performed to full depth as long asthe lordotic curve is maintained. The alignment of the pelvis is correlated with spine curvature and it has also been found to influence lifting function, withan anterior tilt of thepelvis providing increased trunk muscle activity. ${ }^{10}$ The majority of research on squat technique provide no quantified measure or description of the pelvic tilt. Therefore, the purpose of the present study was to measure the effect of squat amplitude on pelvictilt and tibia inclination.

\section{Materials and methods}

\section{Participants}

Eighteen male subjects (age: $26 \pm 6$ years, height: $178 \pm 7 \mathrm{~cm}$, total body mass: $81.3 \pm 11 \mathrm{~kg}$, resistance training experience: $5 \pm 2$ years) were evaluated. Subjects had no previous lower back injury, surgery in the lower extremities, and no history of injury with residual symptoms (pain, "giving-away" sensations) in the lower limbs within the last year. This study was approved by the University research ethics committee and all subjects read and signed an informed consent document (\#68/2016).

\section{Procedures}

Subjects were instructed in properisometric back squat technique for both conditions (partial: at $90^{\circ}$ knee flexion, and full: at $140^{\circ}$ knee flexion). Knee angle was measured by a goniometer. Their feet were positioned at hip width and vertically aligned with the barbell. The barbell was positioned on the shoulders (high-bar position) and all subjects performed each isometric squat condition three times for 3-s (rest between reps?). During each squat, the degree of pelvic tilt and tibial inclination were measured, and the highest value was used. Pelvictilt and tibial inclination were measured by a digital inclinometer (Max Measure, USA, accuracy: $\pm 0.02^{\circ}$, resolution: $0.05^{\circ}$ ) fixed on the sacrum and on the tibia, at an orthostatic position with a neutral spine. For pelvic tilt, positive values refer to anterior/forward and negative to posterior/backward positions. A rest period of 5-min was provided between conditions. All measures were performed at the same hour of the day, between 5 and 7 PM, and by the same researcher. A paired student t-test and a significance of 5\% was used.Cohen's formula for effect size $(d)$ was calculated, and the results were based on the following criteria: $<0.35$ trivial effects; $0.35-0.80$ small effect; 0.80 1.50 moderate effect; and $>1.5$ large effect, for recreationally trained subjects. ${ }^{11}$

\section{Results and discussion}

There were significant differences in pelvic tilt between partial and full amplitudes $\left(+32.4^{\circ} \pm 10.9\right.$ and $-21.7^{\circ} \pm 12.3$, respectively, $P<0.001, d=0.95, \Delta \%=33.8 \%$ ) (Figure 1). Maximum tibial inclination values did not show significant differences between partial and full amplitudes $\left(19.1^{\circ} \pm 6.6\right.$ and $20.1^{\circ} \pm 7.4$, respectively, $P=0.225, d=0.14$, $\Delta \%=4,9 \%)$. 


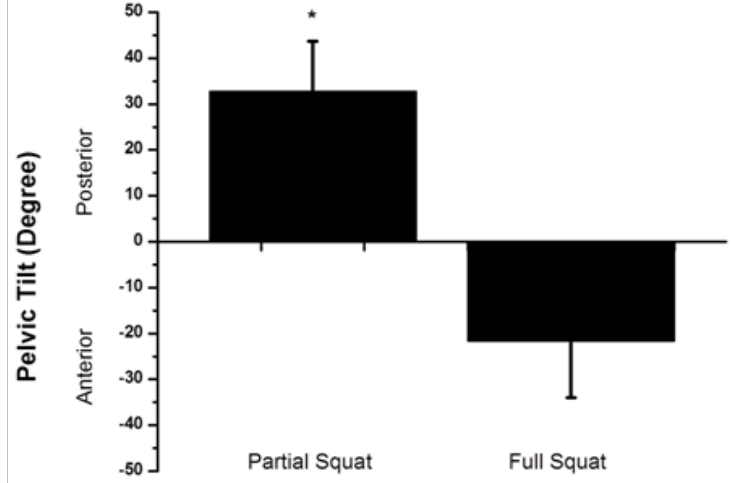

Figure I Mean and standard deviation of pelvic tilt in different back squat conditions (partial and full amplitude). $* P<0.05$.

The present results demonstrate important differences between partial and full squats based on pelvic tilt. During the partial squat, the pelvis hadan an terior tilt, increasing the lordotic position, while the full squat moved the pelvis backward creating lumbar retification.

The back musculature supports the spine in a neutral position. Increased and potentially harmful compressive and shear forces of the lumbar spine may result during intense squat conditions. ${ }^{12}$ Therisk of disc herniation is increased during heavy resistance squatting, with both the flexed spine position, and the backward pelvis tilt as a resultof excessive stress placed on intervertebral discs. ${ }^{13}$

Spinal flexion and extension have been shown to significantly impact joint kinetics during squat performance. Squattin gwith a flexed lumbar spine decreases the moment arm for the lumbar erector spinae, reduces tolerance to compressive load, and results in a transfer of the load from muscles to passive tissues, heightening the risk of disc herniation. Moreover, shear forces during squatting have been found to be significantly greater as lumbar flexion increases from the neutral position. ${ }^{12}$

Previous studies have shown that compressive forces increase during excessive lumbar extension. ${ }^{14-17}$ Therefore, it is advisable to maintain a neutral spine throughout performance of the squat, avoiding any excessive flexion or extension. Furthermore, the lack of tibial inclination differences demonstrates that it does not represent a major influence on control of the center of mass during both squat amplitudes

\section{Conclusion}

The partial squat produces anterior pelvic tilt, while the full squat produces backward pelvic tilt. Inclination of the tibia is similar in both amplitudes of the squat.

\section{Acknowledgements}

None.

\section{Conflict of interest}

Authors declares there is no conflict of interest in publishing the article

\section{References}

1. Contreras B, Vigotsky AD, Schoenfeld BJ, et al. A Comparison of Gluteus Maximus, Biceps Femoris, and Vastus Lateralis Electromyography Amplitude in the Parallel, Full, and Front Squat Variations in Resistance Trained Females. J Appl Biomech. 2016;32(1):16-22.

2. Da Silva JJ, Schoenfeld BJ, Marchetti PN, et al. Muscle Activation Differs Between Partial and Full Back Squat Exercise with External Load Equated. J Strength Cond Res. 2017.

3. Marchetti PH, Da Silva JJ, Schoenfeld BJ, et al. Muscle Activation Differs between Three Different Knee Joint-Angle Positions during a Maximal Isometric Back Squat Exercise. J Sports Med (Hindawi Publ Corp). 2016;2016:3846123.

4. McKean MR, Dunn PK, Burkett BJ. The lumbar and sacrum movement pattern during the back squat exercise. J Strength Cond Res. 2010;24(10):2731-2741.

5. Caterisano A, Moss RF, Pellinger TK, et al. The effect of back squat depht on the EMG activity of 4 superficial hip and thigh muscles. $J$ Strength Cond Res. 2002;16(3):428-432.

6. Comfort P, Kasim P. Optimizing squat technique. Strength Cond J. 2007;29(6):10-13.

7. Myer GD, Kushner AM, Brent JL, et al. The Back Squat: A Proposed Assessment of Functional Deficits and Technical Factors That Limit Performance. Strength and Conditioning Journal. 2014;36(6):4-27.

8. McCaw ST, Melrose DR. Stance width and bar load effects on leg muscle activity during the parallel squat. Med Sci Sports Exerc. 1999;31(3):428-436.

9. Lindbeck L, Kjellberg K. Gender differences in lifting technique. Ergonomics. 2001;44(2):202-214.

10. Delitto RS, Rose SJ. An electromyographic analysis of two techniques for squat lifting and lowering. J Phys Ther. 1992;72(6):438-448.

11. Rhea MR. Determining the magnitude of treatment effects in strength training research through the use of the effect size. J Strength Cond Res. 2004;18(4):918-920.

12. Schoenfeld BJ. Squatting kinematics and kinetics and their application to exercise performance. J Strength Cond Res. 2010;24(12):3497-3506.

13. Hemmerich A, Brown $\mathrm{H}$, Smith $\mathrm{S}$, et al. Hip, knee, and ankle kinematics of high range of motion activities of daily living. J Orthop Res. 2006;24(4):770-781.

14. Adams MA, May S, Freeman BJ, et al. Effects of backward bending on lumbar intervertebral discs: Relevance to physical therapy treatments for low back pain. Spine (Phila Pa 1976). 2000;25(4):431-437.

15. Adams MA, Dolan P. Recent advances in lumbar spine mechanics and their clinical significance. Clin Biomech Clin Biomech (Bristol, Avon). 1995;10(1):3-19.

16. Contreras B, Vigotsky AD, Schoenfeld BJ, et al. A Comparison of Gluteus Maximus, Biceps Femoris, and Vastus Lateralis Electromyographic Activity in the Back Squat and Barbell Hip Thrust Exercises. $J$ Appl Biomech. 2015;31(6):452-458.

17. Donnelly DV, Berg WP, Fiske DM. The effect of the direction of gaze on the kinematics of the squat exercise. J Strength Cond Res 2006;20(1):145-150 